\title{
Predictors of Mortality among United States Veterans with Human Immunodeficiency Virus and Hepatitis C Virus Coinfection
}

\author{
Sebhat Erqou, ${ }^{1,2}$ Arpan Mohanty, ${ }^{3}$ Pashtoon Murtaza Kasi, ${ }^{4}$ and Adeel A. Butt ${ }^{2,5,6}$ \\ ${ }^{1}$ Department of Medicine, Division of Hospital Medicine, Weill Cornell Medical College, 525 East 68th Street, \\ P.O. Box 130, New York, NY 10065, USA \\ ${ }^{2}$ VA Pittsburgh Health Care System, Pittsburgh, PA 15213, USA \\ ${ }^{3}$ Department of Medicine, Yale School of Medicine, New Haven, CT 06510, USA \\ ${ }^{4}$ Department of Medicine, Mayo Clinic, Rochester, MN 55905, USA \\ ${ }^{5}$ Department of Medicine, Sheikh Khalifa Medical City, Abu Dhabi, UAE \\ ${ }^{6}$ Department of Medicine, University of Pittsburgh School of Medicine, Pittsburgh, PA 15213, USA
}

Correspondence should be addressed to Sebhat Erqou; see9003@med.cornell.edu

Received 4 January 2014; Accepted 6 February 2014; Published 7 April 2014

Academic Editors: L. Rodrigo and H. Yoshiji

Copyright ( 2014 Sebhat Erqou et al. This is an open access article distributed under the Creative Commons Attribution License, which permits unrestricted use, distribution, and reproduction in any medium, provided the original work is properly cited.

\begin{abstract}
Background. Understanding the predictors of mortality in individuals with human immunodeficiency virus and hepatitis $C$ virus (HIV/HCV) coinfection can be useful in management of these patients. Methods. We used the Electronically Retrieved Cohort of HCV Infected Veterans (ERCHIVES) for these analyses. Multivariate Cox-regression models were used to determine predictors of mortality. Results. Among 8,039 HIV infected veterans, 5251 (65.3\%) had HCV coinfection. The all-cause mortality rate was 74.1 (70.4-77.9) per 1000 person-years (PY) among veterans with HIV/HCV coinfection and 39.8 (36.3-43.6) per 1000 PY for veterans with HIV monoinfection. The multivariable adjusted hazard ratio ( $95 \%$ confidence interval) of all-cause mortality for HCV infection was 1.58 (1.36-1.84). Positive predictors of mortality included decompensated liver disease (2.33 (1.98-2.74)), coronary artery disease $(1.74(1.32-2.28))$, chronic kidney disease $(1.62(1.36-1.92))$, and anemia (1.58 (1.31-1.89)). Factors associated with reduced mortality included HCV treatment $(0.41(0.27-0.63))$ and higher CD4 count $(0.90(0.87-0.93)$ per 100 cells $/ \mu \mathrm{L}$ higher count). Data were insufficient to make informative analyses of the role of HCV virologic response. Conclusion. HCV coinfection was associated with substantial increased risk of mortality among HIV infected veterans. HCV treatment was associated with significantly lower risk of mortality.
\end{abstract}

\section{Background}

Human immunodeficiency virus (HIV) is an important cause of morbidity and mortality globally. Worldwide, there are approximately 34 million people with HIV infection, $68 \%$ of which are in sub-Saharan African countries [1]. In 2010, there were 1.8 million deaths due to HIV/AIDS globally [1]. In the United States, there were about 1.1 million people infected with HIV in 2010 [2], and 16 thousand individuals died of HIV [2]. Approximately $30-40 \%$ of HIV infected persons are coinfected with hepatitis C virus (HCV) $[3,4]$. This proportion is as high as $70 \%$ among individuals who use intravenous drugs [5].
Prior to the advent of highly active antiretroviral therapy (HAART) the outcome of HIV infected individuals has been largely the function of progression to acquired immunodeficiency syndrome (AIDS) and development of opportunistic infections. In the HAART era, the longevity of HIV infected individuals has significantly improved and chronic conditions such as HCV infection are emerging as important factors in morbidity and mortality [6-8].

$\mathrm{HIV} / \mathrm{HCV}$ coinfected individuals develop the complications of HCV infection (i.e., cirrhosis of liver, end-stage liver disease, and hepatocellular carcinoma) more rapidly than those with HCV monoinfection $[9,10]$. Similarly, some 
studies have shown that the incidence of AIDS defining illnesses are increased among HIV/HCV infected individuals compared to those with HIV monoinfection [11-13], while others have not confirmed this [7, 13]. Regardless of the specific outcomes, it is well established that individuals with $\mathrm{HIV} / \mathrm{HCV}$ coinfection have significantly higher morbidity and mortality compared to individuals with HIV monoinfection $[7,12,13]$. However, specific predictors of mortality and how they differ in the HIV/HCV coinfected versus HIV monoinfected individuals in the era of HAART have not been well documented.

Understanding the predictors of mortality in $\mathrm{HIV} / \mathrm{HCV}$ coinfected individuals can help identify potential targets for interventions that may improve survival. We analyzed data from the Electronically Retrieved Cohort of HCV Infected Veterans (ERCHIVES) study to determine the incidence of all-cause mortality and its predictors among $\mathrm{HIV} / \mathrm{HCV}$ coinfected individuals and HIV monoinfected counterparts.

\section{Patients and Methods}

ERCHIVES is a retrospective cohort of HCV infected individuals and HCV uninfected controls. Version 2 of ERCHIVES, which was used for the current analyses, was assembled and merged from 2001 to 2008 through linkage of records from several sources of the Veterans Health Administration Healthcare System (VA) [14-18]. Demographic and clinical data were retrieved from the Department of Veterans Affairs National Patient Care Database, pharmacy data from the Pharmacy Benefits Management Database, laboratory data from the Decision Support System and the Corporate Data Warehouse, and mortality data from the Beneficiary Identification Records Locator Subsystem. HCV infected individuals were identified by a positive $\mathrm{HCV}$ antibody at baseline. Corresponding HCV uninfected controls were identified by a negative $\mathrm{HCV}$ antibody test performed in the same year as a positive antibody test for the cases and were matched by age (5-year blocks), race, sex, and geographic location. HIV infected individuals were identified by positive HIV antibody test during routine clinical care. For the current study, we included subjects with HIV monoinfection and HIV/HCV coinfection.

Baseline demographics and comorbidities, including smoking, alcohol and drug abuse or dependence, hypertension, diabetes, chronic kidney disease (CKD), decompensated liver disease, chronic obstructive pulmonary disease (COPD), cancer, coronary artery disease (CAD), peripheral vascular disease (PVD), and stroke, were defined as described previously [15-20]. Comorbidities were considered as baseline if first recorded prior to, or within 6 months after, entry to cohort. Patients with decompensated liver disease at baseline were identified using a combination of ICD codes and laboratory parameters including elevated INR $(>1.7)$ or bilirubin $(>2 \mathrm{~g} / \mathrm{dL})$ or lower albumin $(<2.5 \mathrm{~g} / \mathrm{dL})$ concentrations, as previously described [21]. Laboratory data retrieved included hemoglobin, white blood cell count, total cholesterol, low-density and high-density lipoprotein cholesterol, triglycerides, alanine aminotransferase (ALT), aspartate aminotransferase (AST), and HCV and HIV antibody tests. A subset of participants had available information on CD4 counts, HIV RNA levels and/or HCV RNA levels. Anemia was defined as hemoglobin level less than $13 \mathrm{~g} / \mathrm{dL}$ or $12 \mathrm{~g} / \mathrm{dL}$ for men and women, respectively. Due to limitations in data, virologic response was defined as undetectable HCV RNA level on treatment, at the end of treatment, or after treatment, for the purpose of this study.

Data on use of interferon alpha, pegylated interferon alpha, and ribavirin in various combinations was obtained from the Pharmacy Benefits Management Database. Dates of starting treatment and cumulative durations of prescription were obtained. Patients with interferon doses higher than those routinely used for HCV treatment were excluded [22]. All-cause mortality data were obtained from VA Beneficiary Identification Records Locator System. Subjects were followed up until death or the last observation date in the cohort. Individuals with no follow-up visits after baseline visit were excluded from analyses.

Baseline characteristics of HIV/HCV coinfected individuals were compared to those with HIV monoinfection using $t$-test for continuous variables and chi-square test for categorical variables. Survival analyses were used to determine the associations of HCV coinfection status and mortality. The Kaplan-Meier plots were used to visually compare survivor function by HCV status. Log-rank test was used to determine the statistical significance of differences between survivor curves. The hazard ratios (HRs) and 95\% confidence intervals (CIs) for the association of HCV status with mortality among HIV infected individuals were estimated using the Cox proportional hazard models with adjustment for confounders. Predictors of mortality were investigated among individuals with HIV/HCV coinfection and those with HIV monoinfection using the Cox models. Covariates included in the multivariate Cox model were (i) sociodemographic factors including age, sex, race, smoking, alcohol, and drug abuse, (ii) baseline comorbidities, including diabetes, hypertension, decompensated liver disease, CKD, COPD, cancer, CAD, stroke, and PVD, and (iii) CD4 count, HIV RNA level, and HCV treatment status. The assumptions of the proportionality of hazards were evaluated using the Schoenfeld residuals. All analyses were performed using Stata (College Station, TX), version 9.

\section{Results}

The baseline characteristics of the participants are shown in Table 1. Among 8,039 HIV infected veterans, 5251 (65.3\%) had HCV coinfection. The mean (SD) age of participants was 50.0 (7.3) years; $98.2 \%$ were male, $29.5 \%$ White, $57.9 \%$ Black, and 7.4\% Hispanic. Participants with HIV/HCV coinfection, compared to those with HCV monoinfection, were more likely to be Black ( $60 \%$ versus $54 \%$ ) or smokers ( $20 \%$ versus $17 \%)$, have hypertension (32\% versus $29 \%$ ), diabetes ( $14 \%$ versus $11 \%$ ), anemia (52 versus $44 \%$ ), or decompensated liver disease (26\% versus $20 \%$ ), or have a history of drug abuse or dependence $(39 \%$ versus $18 \%$ ) or alcohol abuse or dependence $(28 \%$ versus $16 \%)$ ( $P$ value $<0.01$, for all 
TABLE 1: Baseline characteristics of HIV infected veterans included in analyses, by HCV status.

\begin{tabular}{|c|c|c|c|c|c|}
\hline \multirow{2}{*}{ Variable } & \multicolumn{2}{|c|}{$\mathrm{HCV}+$} & \multicolumn{2}{|c|}{$\mathrm{HCV}-$} & \multirow{2}{*}{$P$ value } \\
\hline & Number available & Mean (SD) or $N(\%)$ & Number available & Mean $(\mathrm{SD})$ or $N(\%)$ & \\
\hline Age (yrs) & 5251 & $50(7)$ & 2788 & $49(7)$ & $<0.0001$ \\
\hline Male & 5251 & $5146(98 \%)$ & 2788 & $2745(98 \%)$ & 0.15 \\
\hline White & 5251 & $1398(27 \%)$ & 2788 & $975(35 \%)$ & \\
\hline Black & 5251 & $3146(60 \%)$ & 2788 & $1510(54 \%)$ & $<0.0001$ \\
\hline Hispanic & 5251 & $435(8 \%)$ & 2788 & $160(6 \%)$ & \\
\hline Smoking & 5251 & $1068(20 \%)$ & 2788 & $480(17 \%)$ & 0.001 \\
\hline Hypertension & 5251 & $1674(32 \%)$ & 2788 & $800(29 \%)$ & 0.003 \\
\hline Diabetes & 5251 & $729(14 \%)$ & 2788 & $297(11 \%)$ & $<0.0001$ \\
\hline CKD & 5251 & $1100(21 \%)$ & 2788 & $520(19 \%)$ & 0.015 \\
\hline COPD & 5251 & $404(8 \%)$ & 2788 & $167(6 \%)$ & 0.005 \\
\hline Anemia & 5251 & $2718(52 \%)$ & 2788 & $1215(44 \%)$ & $<0.0001$ \\
\hline CAD & 5251 & $213(4 \%)$ & 2788 & $104(4 \%)$ & 0.48 \\
\hline PVD & 5251 & $97(2 \%)$ & 2788 & $41(1 \%)$ & 0.22 \\
\hline Stroke & 5251 & $68(1 \%)$ & 2788 & $34(1 \%)$ & 0.77 \\
\hline Cancer or history & 5251 & $474(9 \%)$ & 2788 & $291(10 \%)$ & 0.04 \\
\hline DLD & 4279 & $1093(26 \%)$ & 2347 & $465(20 \%)$ & $<0.0001$ \\
\hline Drug abuse & 5251 & $2052(39 \%)$ & 2788 & $513(18 \%)$ & $<0.0001$ \\
\hline Alcohol abuse & 5251 & $1451(28 \%)$ & 2788 & $434(16 \%)$ & $<0.0001$ \\
\hline Major depression & 5251 & $561(11 \%)$ & 2788 & $269(10 \%)$ & 0.15 \\
\hline BMI $\left(\mathrm{kg} / \mathrm{m}^{2}\right)$ & 5143 & $24.7(5.7)$ & 2743 & $25.4(4.5)$ & $<0.0001$ \\
\hline CD4 count (cells/uL) & 3582 & $355(290)$ & 1972 & $366(296)$ & 0.15 \\
\hline HIV RNA (copies/uL) & 2745 & $69812(123540)$ & 1379 & 70143 (128887) & 0.94 \\
\hline Leukocyte count (cells/uL) & 4973 & $5.5(2.5)$ & 2556 & $5.7(2.5)$ & 0.0005 \\
\hline AST (IU/L) & 4996 & $70(138)$ & 2637 & $47(115)$ & $<0.0001$ \\
\hline ALT (IU/L) & 4979 & $64(104)$ & 2611 & $46(120)$ & $<0.0001$ \\
\hline Albumin (g/dL) & 4818 & $3.60(0.68)$ & 2447 & $3.87(0.69)$ & $<0.0001$ \\
\hline
\end{tabular}

ALT: alanine transaminase; AST: aspartate transaminase; BMI: body mass index; CAD: coronary artery disease; CKD: chronic kidney disease; COPD: chronic obstructive pulmonary disease; DLD: decompensated liver disease; PVD: peripheral vascular disease.

comparisons). They were also more likely to have lower body mass index $\left(24.7\right.$ versus $\left.25.4 \mathrm{Kg} / \mathrm{m}^{2}\right)$, lower levels of albumin (3.6 versus $3.87 \mathrm{~g} / \mathrm{dL})$, or higher levels of AST (70 versus $47 \mathrm{IU} / \mathrm{L})$ or ALT $(64$ versus $46 \mathrm{IU} / \mathrm{L})(P$ value $<0.001$, for all comparisons). Of note, the groups were similar with regard to CD 4 count (355 versus 366 cells $/ \mu \mathrm{L}, P$ value $=0.15$ ) and HIV viral load (69800 versus $70100 \mathrm{copies} / \mu \mathrm{L}, P$ value $=0.94)$.

The all-cause mortality rate was higher among veterans with HIV/HCV coinfection (HR (95\% CI), 74.1 (70.4-77.9) per 1000 person-years (PY)), compared to those with HIV monoinfection (39.8 (36.3-43.6) per $1000 \mathrm{PY}$ ) (Figure 1). The HR of all-cause mortality for HCV infection was 1.78 (95\% CI, 1.59-1.96) in the age- and sex-adjusted Cox model. The association of HCV with mortality was attenuated by $7 \%$ after adjusting for baseline decompensated liver disease (1.66 (1.44-1.93)). The corresponding HR after adjusting for several baseline sociodemographic and comorbidity variables was 1.58 (1.36-1.84) (Table 2). The association was further reduced to $1.50(1.28-1.75)$ after adjustment for incident cirrhosis. The association of HCV with mortality was 1.47 (1.21-1.79) after adjustment for baseline HIV RNA level in a subset with available data (Supplementary Table 1 in Supplementary Material

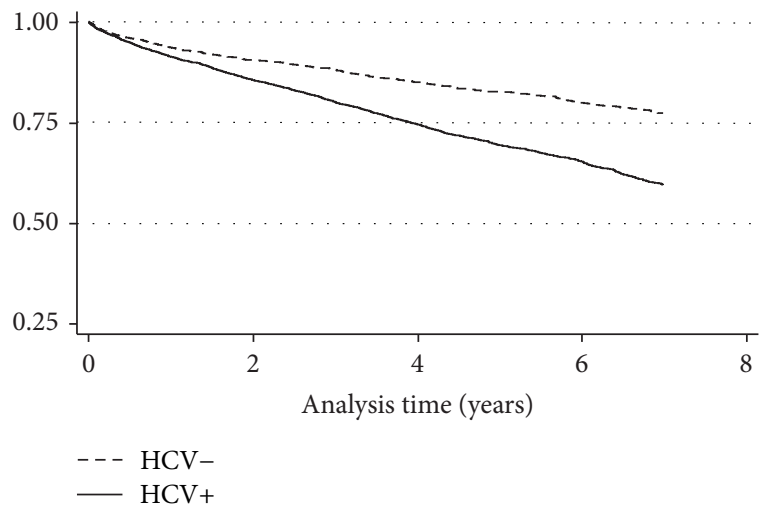

FIGURE 1: The Kaplan-Meier curve for HIV infected veterans by $\mathrm{HCV}$ infection status.

available online at http://dx.doi.org/10.1155/2014/764540). Other baseline variables associated with mortality in the multivariable Cox model among veterans with HIV infection (with or without $\mathrm{HCV}$ coinfection) included decompensated 
TABLE 2: Association of HCV status with mortality among HIV infected veterans in a multivariable regression model.

\begin{tabular}{|c|c|c|c|}
\hline Variables & $\begin{array}{c}\text { Model } 1 \\
N=8030\end{array}$ & $\begin{array}{c}\text { Model } 2 \\
N=4521 \\
\end{array}$ & $\begin{array}{c}\text { Full model } \\
N=4521\end{array}$ \\
\hline $\mathrm{HCV}$ & $1.78(1.59,1.96)$ & $1.66(1.44,1.93)$ & $1.58(1.36,1.84)$ \\
\hline Age (per 10 yrs) & $1.60(1.51,1.70)$ & $1.51(1.40,1.64)$ & $1.32(1.21,1.44)$ \\
\hline Male & $1.00(0.70,1.43)$ & $1.07(0.60,1.89)$ & $0.98(0.55,1.73)$ \\
\hline DLD & - & $3.73(3.29,4.24)$ & $2.17(1.88,2.5)$ \\
\hline Black versus white & - & - & $0.86(0.74,1)$ \\
\hline Hispanic versus white & - & - & $0.79(0.61,1.03)$ \\
\hline BMI $\left(\mathrm{kg} / \mathrm{m}^{2}\right)$ & - & - & $0.94(0.92,0.95)$ \\
\hline Smoking & - & - & $0.97(0.82,1.14)$ \\
\hline Hypertension & - & - & $0.98(0.85,1.14)$ \\
\hline Diabetes & - & - & $1.45(1.22,1.71)$ \\
\hline COPD & - & - & $1.44(1.19,1.75)$ \\
\hline Anemia & - & - & $1.59(1.36,1.86)$ \\
\hline CKD & - & - & $1.74(1.5,2.02)$ \\
\hline CAD & - & - & $1.78(1.42,2.23)$ \\
\hline Stroke & - & - & $1.28(0.87,1.89)$ \\
\hline PVD & - & - & $0.82(0.54,1.23)$ \\
\hline Thromboembolism & - & - & $0.44(0.11,1.79)$ \\
\hline Cancer & - & - & $1.5(1.26,1.79)$ \\
\hline Drug abuse & - & - & $1.06(0.9,1.26)$ \\
\hline Alcohol abuse & - & - & $1.14(0.96,1.35)$ \\
\hline Major depression & - & - & $1.01(0.82,1.24)$ \\
\hline CD4 count (per 100 clls/uL) & - & - & $0.9(0.88,0.93)$ \\
\hline
\end{tabular}

Model 1: 8030 individuals with HIV infection had available data on HCV status, age, and sex.

Model 2: 4521 individuals with HIV infection had available data on HCV status, age, sex, and decompensated liver disease.

Model 3: 4521 individual with HIV infection had available data on all the 22 variables included in model.

ALT: alanine transaminase; AST: aspartate transaminase; BMI: body mass index; CAD: coronary artery disease; CKD: chronic kidney disease; COPD: chronic obstructive pulmonary disease; DLD: decompensated liver disease; PVD: peripheral vascular disease.

liver disease (2.17 (1.88-2.50)), CAD (1.78 (1.42-2.23)), CKD (1.74 (1.50-2.02)), anemia (1.59 (1.36-1.86)), and cancer (1.50 $(1.26-1.69))$. By contrast, higher CD4 count $(0.90(0.88-0.93)$ per 100 cells $/ \mu \mathrm{L}$ higher count) and body mass index (0.94 $(0.92-0.95)$ per $\left.1 \mathrm{~kg} / \mathrm{m}^{2}\right)$ were associated with reduced mortality (Table 2). For comparison, the univariate association of these factors is shown in Supplementary Table 2.

Among veterans with $\mathrm{HIV} / \mathrm{HCV}$ coinfection, decompensated liver disease (HR (95\% CI) 2.33 (1.98-2.74)), CAD (1.74 (1.32-2.28)), CKD (1.62 (1.36-1.92)), anemia (1.58 (1.31-1.89)), COPD (1.57 (1.26-1.95)), cancer (1.52 (1.23-1.87)), and age (1.34 (1.21-1.49) for every 10-year-older age) were predictors of higher risk of mortality, while HCV treatment (0.41 (0.27$0.63))$, CD4 count $(0.90(0.87-0.93)$ per 100 cells $\mu \mathrm{L}$ higher count), and Black race $(0.72(0.60-0.87))$ were associated with significantly lower risk of mortality (Table 3 ). The associations were broadly similar among individuals with HIV monoinfection, except for stroke and diabetes which were strongly associated with mortality, while Hispanic race, thromboembolism, PVD, and drug abuse were inversely associated with mortality, in this group. Unlike in HIV/HCV coinfected veterans, COPD was not associated with mortality in those with monoinfection. For comparison, the univariate association of these factors by HCV status is shown in Supplementary Table 3. We explored the role of $\mathrm{HCV}$ virologic response in a subset of participants that had available data on HCV viral load (Table 4). Of the 469 people that had received HCV treatment, 137 (29\%) had available data on HCV viral load, of which 21 (15\%) had virologic response. Mortality rate was significantly reduced among individuals who received HCV treatment even when they did not achieve virologic response. The number of participants with virologic response was too small to make informative analysis of the association in this category (Table 4).

\section{Discussion}

In our present analyses involving over 8,000 HIV infected veterans, including $65 \%$ with $\mathrm{HCV}$ coinfection, we found a $75 \%$ increased risk of mortality among HIV/HCV coinfected veterans compared to those with HIV monoinfection. Decompensated liver disease, CAD, CKD, and anemia were associated with increased risk of mortality among individuals with HIV/HCV coinfection, while higher CD4 count and HCV treatment were associated with lower risk of mortality in this group. 
TABLE 3: Multivariate predictors of mortality in HIV infected individuals by HCV status.

\begin{tabular}{lcc}
\hline Variables & $\mathrm{HCV}+$ & $\mathrm{HCV}-$ \\
\hline Age (per 10 yrs) & $1.34(1.21,1.49)$ & $1.2(1,1.45)$ \\
Male & $1.02(0.52,1.98)$ & $0.69(0.22,2.18)$ \\
Black versus white & $0.72(0.6,0.87)$ & $1.35(1,1.83)$ \\
Hispanic versus white & $0.82(0.62,1.08)$ & $0.38(0.15,0.95)$ \\
BMI & $0.95(0.94,0.97)$ & $0.89(0.86,0.92)$ \\
Smoking & $0.91(0.75,1.1)$ & $1.19(0.84,1.69)$ \\
Hypertension & $0.96(0.8,1.14)$ & $1.24(0.92,1.66)$ \\
Diabetes & $1.25(1.02,1.52)$ & $2.33(1.67,3.26)$ \\
DLD & $2.33(1.98,2.74)$ & $2.09(1.57,2.8)$ \\
COPD & $1.57(1.26,1.95)$ & $0.91(0.58,1.43)$ \\
Anemia & $1.58(1.31,1.89)$ & $1.49(1.08,2.07)$ \\
CKD & $1.62(1.36,1.92)$ & $1.99(1.47,2.71)$ \\
CAD & $1.74(1.32,2.28)$ & $1.81(1.19,2.73)$ \\
Stroke & $0.99(0.6,1.64)$ & $2.86(1.48,5.53)$ \\
PVD & $0.98(0.63,1.53)$ & $0.33(0.12,0.92)$ \\
Thromboembolism & $0.79(0.11,5.75)$ & $0.1(0.01,0.8)$ \\
Cancer & $1.52(1.23,1.87)$ & $1.55(1.11,2.18)$ \\
Drug abuse & $1.2(1,1.43)$ & $0.57(0.36,0.9)$ \\
Alcohol abuse & $1.12(0.93,1.35)$ & $1.05(0.67,1.65)$ \\
Major depression & $1.04(0.82,1.31)$ & $1.06(0.66,1.71)$ \\
CD4 count (per 100 clls/uL) & $0.9(0.87,0.93)$ & $0.92(0.86,0.97)$ \\
HCV treatment & $0.41(0.27,0.63)$ & - \\
\hline ALT & & -1660 \\
\hline
\end{tabular}

ALT: alanine transaminase; AST: aspartate transaminase; BMI: body mass index; CAD: coronary artery disease; CKD: chronic kidney disease; COPD: chronic obstructive pulmonary disease; DLD: decompensated liver disease; PVD: peripheral vascular disease.

TABLE 4: Association of HCV treatment with mortality by virologic response status in multivariate model.

\begin{tabular}{lcc}
\hline Category & $N$ & HR $(95 \% \mathrm{CI})^{*}$ \\
\hline No HCV treatment & 4,790 & Ref. \\
Treated-no VR & 116 & $0.40(0.16-0.96)$ \\
Treated-VR & 21 & $0.45(0.06-3.20)$ \\
No HCV RNA data & 332 & $0.41(0.25-0.68)$ \\
\hline
\end{tabular}

VR: virologic response.

${ }^{*}$ HRs were similarly adjusted as in Table 3 .

In the pre-HAART era, the high rate of AIDS related mortality had masked the morbidity and mortality associated with HCV in HIV infected individuals. With the advent of HAART and improvement in the longevity of HIV infected individuals, HCV infection has emerged as an important cause of morbidity and mortality $[4,7,12,13,23]$. Several studies assessing individuals with HIV infection in the postHAART era found a 1.4- to 2.5 -fold increased risk of mortality compared with individuals with HIV monoinfection [7, 11, 12]. This is because HIV infection accelerates the course of HCV infection resulting in more rapid progression to cirrhosis and end-stage liver disease $[4,12,24]$. In addition, it has been proposed that the increased risk of cardiovascular and chronic kidney diseases in HCV may be mediated by the extrahepatic manifestations of hepatitis such as glomerulonephritis, insulin resistance, and cryoglobulinemia [23, 25]. On the other hand, whether HCV infection accelerates the course of HIV disease is unclear, with studies reporting conflicting findings [13, 26].

Consistent with previous reports we found substantially increased risk of mortality among HIV/HCV coinfected veterans compared to those with HIV monoinfection. HIV/HCV coinfected veterans had significantly higher prevalence of baseline comorbidities including decompensated liver disease, CKD, and anemia. The association was considerably attenuated with adjustment for several variables, in particular for decompensated liver disease. However, there was still a $60 \%$ highly statistically significant increased risk remaining in the fully adjusted model including 21 variables, indicating that confounding is not likely to explain the observed association. Further adjustment for incident cirrhosis reduced the association only by $10 \%$, which suggests that the increased mortality in HIV/HCV coinfection may not be only mediated by increased progression to end-stage liver disease. We, however, did not have data on cause-specific mortality and hence were not able to explore this hypothesis further.

Understanding the predictors of mortality in individuals with HIV/HCV coinfection can be useful for targeting interventions that may help improve outcome. In the present study, we found several baseline comorbidities to be important predictors of mortality in HIV/HCV coinfected veterans. Decompensated liver disease, CKD, CAD, anemia, COPD, and cancer were each associated with $50-70 \%$ higher risk of death. On the other hand, higher CD4 count and body mass index and HCV treatment were associated with lower risk of mortality in this group. These findings highlight the role of antiretroviral therapy, HCV treatment, and improved nutrition in improving outcomes in $\mathrm{HIV} / \mathrm{HCV}$ coinfected individuals. In particular, the $60 \%$ risk reduction noted for $\mathrm{HCV}$ treatment reinforces its importance in improving the survival of these patients [13]. The importance of this finding is heightened by the fact that about a third of the HIV infected patients are also coinfected with $\operatorname{HCV}[3,4]$. In our study, HCV treatment was associated with reduced mortality in those without virologic response and those without sufficient data to enable determination of the status of virologic response. The later observation may be because these individuals had achieved sustained virologic response or it could be that HCV treatment, regardless of virologic response status, improves survival. For instance, we have previously reported that even incomplete $\mathrm{HCV}$ treatment leads to survival advantage [14].

The strengths of the current report include the large size, involving $8000 \mathrm{HIV}$ infected individuals of whom 5000 had HCV coinfection. Second, availability of a wide range of covariates including several baseline comorbidities enabled good control for confounding in assessing the association of $\mathrm{HCV}$ with mortality in this population. Third, the availability of information on several baseline comorbidity variables also allowed extensive investigation of predictors of mortality in 
HIV/HCV coinfected individuals. Fourth, use of mortality as an endpoint reduced the likelihood of any random or systematic misclassification.

Study limitations include analysis of administrative databases and the fact that data were not collected for the specific purpose of this study. Second, as we used all-cause mortality (as compared to liver related mortality), we were not able to determine the proportion of deaths directly attributable to HCV infection. Third, the predominantly male population of veterans with high burden of comorbidities may be somewhat different from other HIV/HCV coinfected populations, which might limit extrapolation to other settings. Fourth, we did not have sufficient HCV RNA data to make informative analysis of the role of virologic response in predicting mortality among individuals that received HCV treatment. Nonetheless, using data on a large number of HIV/HCV coinfected individuals for whom several baseline and follow-up variables were available, our study provides evidence of the importance of HCV coinfection in HIV infected patients and the role of HCV treatment and appropriate management of medical comorbidities in this population.

In conclusion, we found that $\mathrm{HCV}$ coinfection was associated with substantial increased risk of mortality in HIV infected veterans. The association persisted after taking into account several baseline comorbidity variables and incident cirrhosis. Decompensated liver disease, CAD, CKD, and anemia were associated with materially increased risk of mortality among individuals with HIV/HCV coinfection, while higher CD4 count and HCV treatment were associated with lower risk of mortality in this group. Our findings highlight the need for close monitoring of HIV/HCV coinfected patients with focus on providing them with HCV treatment and appropriate management of comorbidities, in addition to antiretroviral therapy.

\section{Disclaimer}

The views expressed in this paper are those of the authors and do not necessarily reflect the position or policy of the Department of Veterans Affairs.

\section{Conflict of Interests}

The authors declare that there is no conflict of interests regarding the publication of this paper.

\section{Authors' Contribution}

Drs. Butt and Erqou had full access to all the data in the study and take final responsibility for the decision to submit it for publication. Dr. Erqou generated the idea, analyzed the data, and drafted the paper; Dr. Mohanty generated the idea and critically revised the paper; Dr. Kasi critically revised the paper; Dr. Butt generated the idea, analyzed the data, and critically revised the paper; Dr. Butt is the principal investigator of the ERCHIVES database.

\section{Acknowledgments}

This material is the result of work supported by and with the use of facilities at the VA Pittsburgh Healthcare System and the central data repositories maintained by the VA Information Resource Center, including the National Patient Care Database, Decisions Support System Database, and Pharmacy Benefits Management Database.

\section{References}

[1] UNAIDS, "World AIDS Day Report: The State of AIDS Epidemic," Tech. Rep., 2011.

[2] Center for Disease Control (CDC), "HIV Surveillance Report: Diagnoses of HIV Infection and AIDS in the United States and Dependent Areas," Tech. Rep., vol. 23, 2011.

[3] V. Soriano, M. Sulkowski, C. Bergin et al., "Care of patients with chronic hepatitis $\mathrm{C}$ and HIV co-infection: recommendations from the HIV-HCV International Panel," AIDS, vol. 16, no. 6, pp. 813-828, 2002.

[4] H. K. Monga, M. C. Rodriguez-Barradas, K. Breaux et al., "Hepatitis $\mathrm{C}$ virus infection-related morbidity and mortality among patients with human immunodeficiency virus infection," Clinical Infectious Diseases, vol. 33, no. 2, pp. 240-247, 2001.

[5] G. Verucchi, L. Calza, R. Manfredi, and F. Chiodo, "Human immunodeficiency virus and hepatitis $\mathrm{C}$ virus coinfection: epidemiology, natural history, therapeutic options and clinical management," Infection, vol. 32, no. 1, pp. 33-46, 2004.

[6] R. K. Sterling, E. Lissen, N. Clumeck et al., "Development of a simple noninvasive index to predict significant fibrosis in patients with HIV/HCV coinfection," Hepatology, vol. 43, no. 6, pp. 1317-1325, 2006.

[7] T.-Y. Chen, E. L. Ding, G. R. Seage III, and A. Y. Kim, "Metaanalysis: increased mortality associated with hepatitis $C$ in HIV-infected persons is unrelated to HIV disease progression," Clinical Infectious Diseases, vol. 49, no. 10, pp. 1605-1615, 2009.

[8] L. Martín-Carbonero, V. Soriano, E. Valencia, J. GarcíaSamaniego, M. López, and J. González-Lahoz, "Increasing impact of chronic viral hepatitis on hospital admissions and mortality among HIV-infected patients," AIDS Research and Human Retroviruses, vol. 17, no. 16, pp. 1467-1471, 2001.

[9] B. Soto, A. Sánchez-Quijano, L. Rodrigo et al., "Human immunodeficiency virus infection modifies the natural history of chronic parenterally-acquired hepatitis $\mathrm{C}$ with an unusually rapid progression to cirrhosis," Journal of Hepatology, vol. 26, no. 1, pp. 1-5, 1997.

[10] A. Sanchez-Quijano, J. Andreu, F. Gavilan et al., "Influence of human immunodeficiency virus type 1 infection on the natural course of chronic parenterally acquired hepatitis C," European Journal of Clinical Microbiology and Infectious Diseases, vol. 14, no. 11, pp. 949-953, 1995.

[11] G. Greub, B. Ledergerber, M. Battegay et al., "Clinical progression, survival, and immune recovery during antiretroviral therapy in patients with HIV-1 and hepatitis C virus coinfection: the swiss HIV cohort study," The Lancet, vol. 356, no. 9244, pp. 1800-1805, 2000.

[12] K. B. Anderson, J. L. Guest, and D. Rimland, "Hepatitis C virus coinfection increases mortality in HIV-infected patients in the highly active antiretroviral therapy era: data from the HIV Atlanta VA cohort study," Clinical Infectious Diseases, vol. 39, no. 10, pp. 1507-1513, 2004. 
[13] L. Piroth, "Coinfection with hepatitis c virus and HIV: more than double trouble," Clinical Infectious Diseases, vol. 49, no. 4 , pp. 623-625, 2009.

[14] A. A. Butt, X. Wang, and C. G. Moore, "Effect of hepatitis C virus and its treatment on survival," Hepatology, vol. 50, no. 2, pp. 387-392, 2009.

[15] A. A. Butt, A. C. Justice, M. Skanderson, M. O. Rigsby, C. B. Good, and C. K. Kwoh, "Rate and predictors of treatment prescription for hepatitis C," Gut, vol. 56, no. 3, pp. 385-389, 2007.

[16] A. A. Butt, K. A. McGinnis, M. Skanderson, and A. C. Justice, "Hepatitis C treatment completion rates in routine clinical care," Liver International, vol. 30, no. 2, pp. 240-250, 2010.

[17] A. A. Butt, U. A. Khan, K. A. McGinnis, M. Skanderson, and C. Kent Kwoh, "Co-morbid medical and psychiatric illness and substance abuse in HCV-infected and uninfected veterans," Journal of Viral Hepatitis, vol. 14, no. 12, pp. 890-896, 2007.

[18] A. A. Butt, X. Wang, and L. F. Fried, "HCV infection and the incidence of CKD," The American Journal of Kidney Diseases, vol. 57, no. 3, pp. 396-402, 2011.

[19] A. A. Butt, K. McGinnis, M. C. Rodriguez-Barradas et al., "HIV infection and the risk of diabetes mellitus," AIDS, vol. 23, no. 10, pp. 1227-1234, 2009.

[20] S. L. Fultz, M. Skanderson, L. A. Mole et al., "Development and verification of a "virtual" cohort using the national VA health information system," Medical Care, vol. 44, no. 8, supplement, pp. S25-S30, 2006.

[21] V. L. Re III, J. K. Lim, M. B. Goetz et al., "Validity of diagnostic codes and liver-related laboratory abnormalities to identify hepatic decompensation events in the Veterans Aging Cohort Study," Pharmacoepidemiology and Drug Safety, vol. 20, no. 7, pp. 689-699, 2011.

[22] M. G. Ghany, D. B. Strader, D. L. Thomas, and L. B. Seeff, "Diagnosis, management, and treatment of hepatitis C: an update," Hepatology, vol. 49, no. 4, pp. 1335-1374, 2009.

[23] E. M. Tedaldi, R. K. Baker, A. C. Moorman et al., "Influence of coinfection with hepatitis $\mathrm{C}$ virus on morbidity and mortality due to human immunodeficiency virus infection in the era of highly active antiretroviral therapy," Clinical Infectious Diseases, vol. 36, no. 3, pp. 363-367, 2003.

[24] V. Di Martino, P. Rufat, N. Boyer et al., "The influence of human immunodeficiency virus coinfection on chronic hepatitis $\mathrm{C}$ in injection drug users: a long-term retrospective cohort study," Hepatology, vol. 34, no. 6, pp. 1193-1199, 2001.

[25] M. J. Koziel and M. G. Peters, "Viral hepatitis in HIV infection," The New England Journal of Medicine, vol. 356, no. 14, pp. 14451454, 2007.

[26] A. d'Arminio Monforte, A. Cozzi-Lepri, A. Castagna et al., "Risk of developing specific aids-defining illnesses in patients coinfected with HIV and hepatitis c virus with or without liver cirrhosis," Clinical Infectious Diseases, vol. 49, no. 4, pp. 612-622, 2009. 


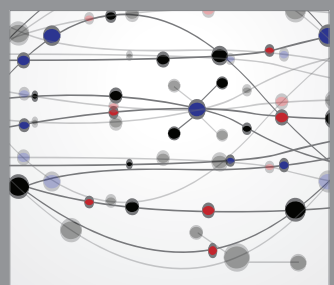

The Scientific World Journal
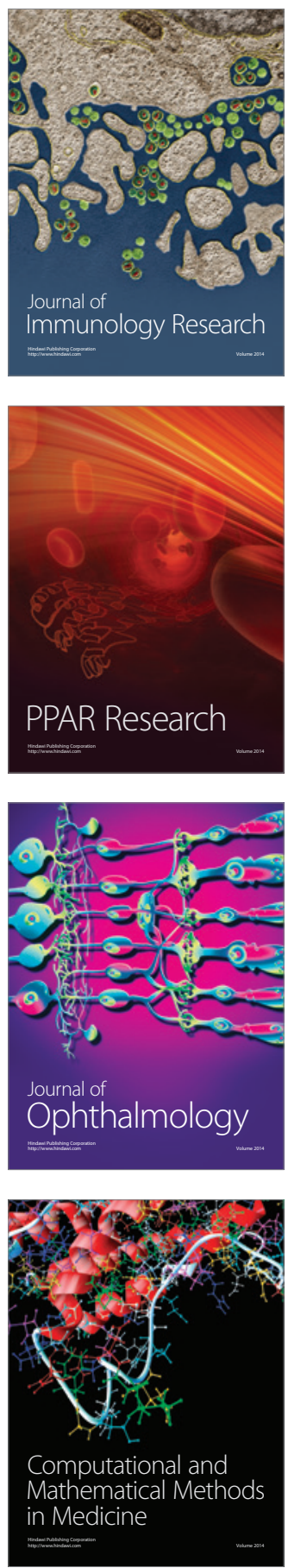

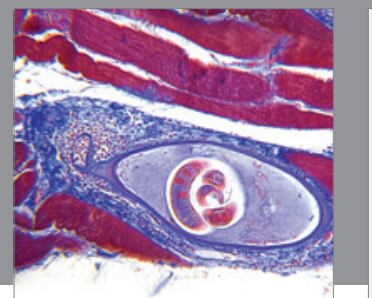

Gastroenterology

Research and Practice
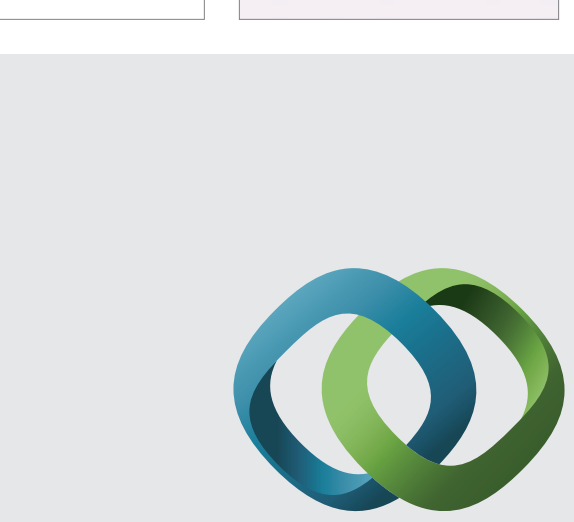

\section{Hindawi}

Submit your manuscripts at

http://www.hindawi.com
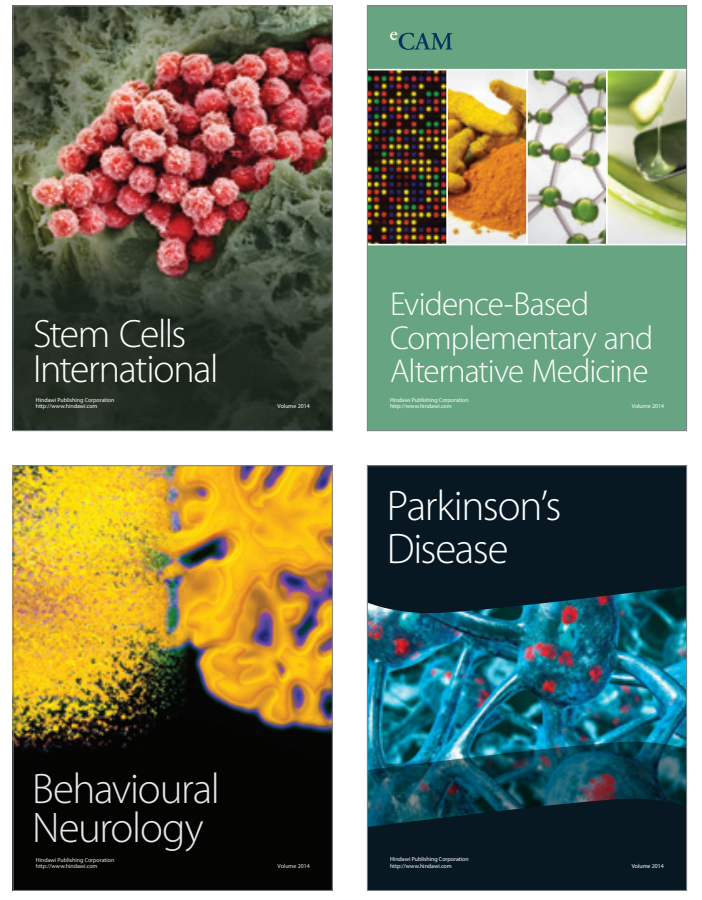
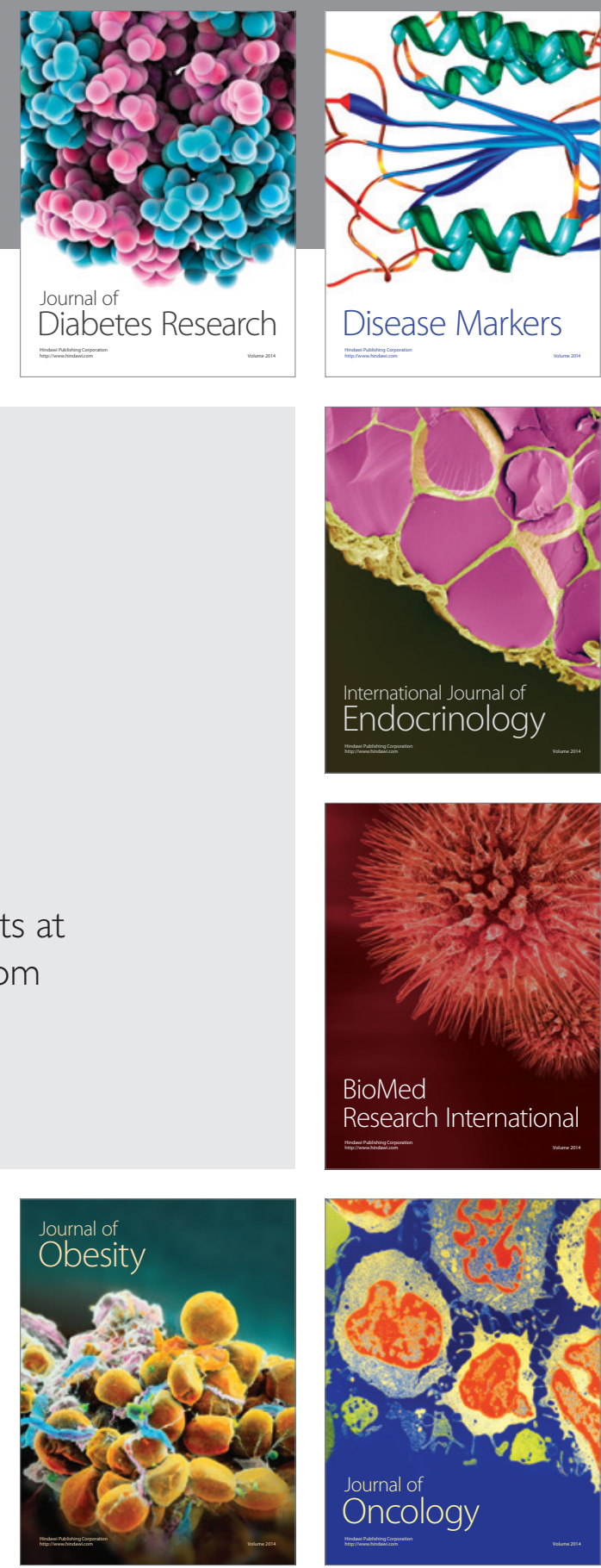

Disease Markers
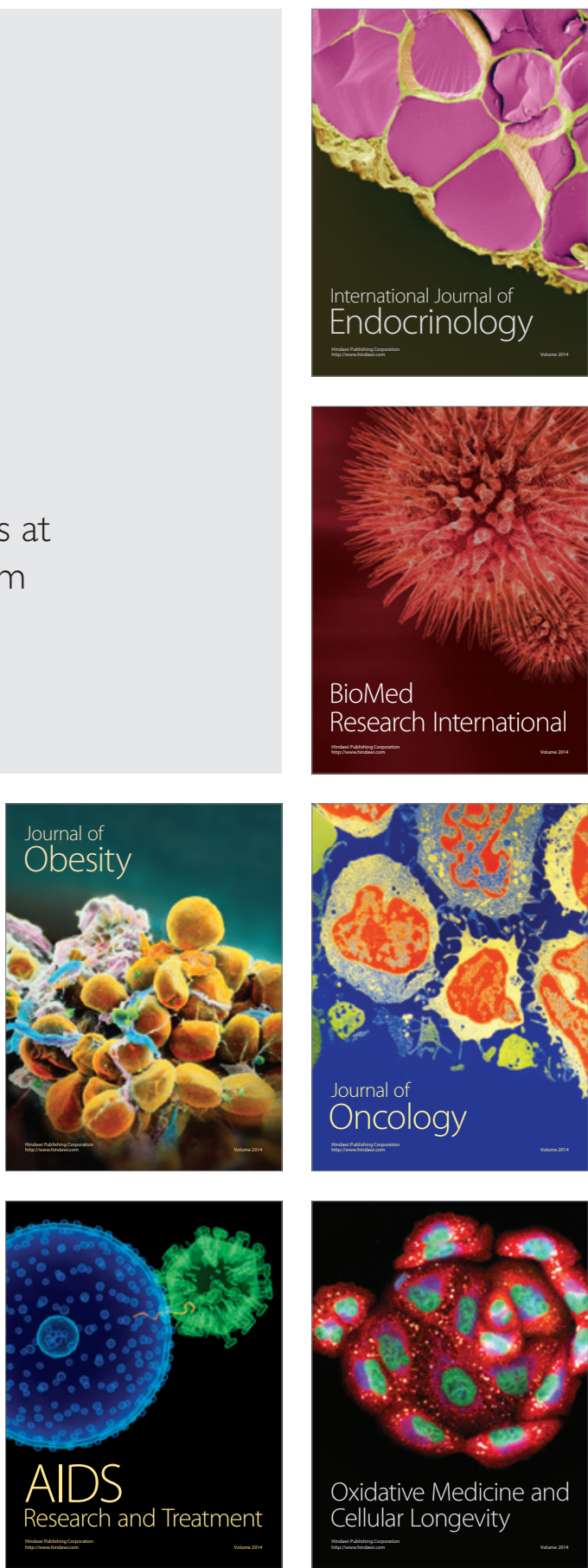